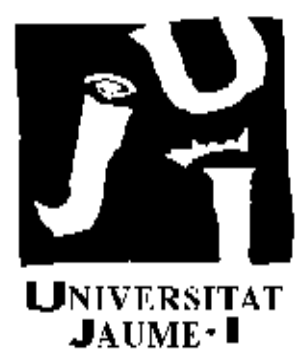

Informe Técnico ICC 02-01-2008

\title{
Attaining High Performance in General-Purpose Computations on Current Graphics Processors
}

Francisco Igual-Peña, Rafael Mayo-Gual, Enrique S. Quintana-Ortí

Enero de 2008

Departamento de Ingeniería y Ciencia de Computadores

Correo electrónico: \{figual, mayo, quintana\}@icc.uji.es

Universidad Jaime I

Campus de Riu Sec, s/n

12.071 - Castellón

España 



\title{
Attaining High Performance in General-Purpose Computations on Current Graphics Processors
}

\author{
Francisco Igual-Peña ${ }^{1}$ \\ Rafael Mayo-Gual ${ }^{2}$, \\ Enrique S. Quintana-Ortî́,
}

\begin{abstract}
:
The increase in performance of the last generations of graphics processors (GPUs) has made this class of hardware a coprocessing platform of remarkable success in certain types of operations. In this paper we evaluate the performance of linear algebra and image processing routines, both on classical and unified GPU architectures and traditional processors (CPUs). From this study, we gain insights on the properties that make an algorithm likely to deliver high performance on a GPU.
\end{abstract}

\section{Keywords:}

Graphics processors (GPUs), general purpose computing on GPU, linear algebra, image processing, high performance.

\footnotetext{
${ }^{1}$ Departamento de Ingeniería y Ciencia de los Computadores E-mail: figual@icc.uji.es.

2 Departamento de Ingeniería y Ciencia de los Computadores E-mail: mayo@icc.uji.es.

${ }^{3}$ Departamento de Ingeniería y Ciencia de los Computadores E-mail: quintana@icc.uji.es.
} 



\title{
Obtención de Altas Prestaciones en Computación de Carácter General sobre Procesadores Gráficos
}

\author{
Francisco Igual-Peña ${ }^{4}$ \\ Rafael Mayo-Gual ${ }^{5}$, \\ Enrique S. Quintana-Ortí ${ }^{6}$,
}

\section{Resumen:}

El aumento de prestaciones de las últimas generaciones de procesadores gráficos (GPUs) ha convertido a este tipo de hardware en una plataforma de coprocesamiento de considerable éxito para cierto tipo de aplicaciones.

En el presente artículo, se evalua el rendimiento de rutinas de álgebra lineal y procesamiento de imágenes, tanto en arquitecuturas gráficas clásicas como unificadas, así como sobre procesadores de carácter general (CPUs).

A partir de dicho estudio, se extraen las propiedades que hacen de un algoritmo candidato a obtener elevado rendimiento al ser ejecutado sobre una GPU.

\section{Palabras clave:}

Procesadores gráficos (GPUs), procesamiento de carácter general sobre GPUs, álgebra lineal, procesamiento de imágenes, altas prestaciones.

\footnotetext{
${ }^{4}$ Departamento de Ingeniería y Ciencia de los Computadores E-mail: figual@icc.uji.es.

${ }^{5}$ Departamento de Ingeniería y Ciencia de los Computadores E-mail: mayo@icc.uji.es.

${ }^{6}$ Departamento de Ingeniería y Ciencia de los Computadores E-mail: quintana@icc.uji.es.
} 



\title{
Attaining High Performance in General-Purpose Computations on Current Graphics Processors
}

\author{
Francisco Igual-Peña, Rafael Mayo-Gual, and Enrique S. Quintana-Ortí \\ Depto. Ingeniería y Ciencia de los Computadores, Universidad Jaume I, \\ 12.071-Castellón, Spain, \{figual, mayo, quintana\}@icc.uji.es
}

\begin{abstract}
The increase in performance of the last generations of graphics processors (GPUs) has made this class of hardware a coprocessing platform of remarkable success in certain types of operations. In this paper we evaluate the performance of linear algebra and image processing routines, both on classical and unified GPU architectures and traditional processors (CPUs). From this study, we gain insights on the properties that make an algorithm likely to deliver high performance on a GPU.
\end{abstract}

Key words: Graphics processors (GPUs), general purpose computing on GPU, linear algebra, image processing, high performance.

\section{Introduction}

During the last years, since the emergence of the first generation of programmable graphics processors (GPUs), many studies have evaluated the performance of these architectures on a large number of applications. Thus, linear algebra operations $[11,6]$, medical image processing $[10,15]$, or database querying [8] are just a few examples of different arenas in which GPU computation has been successfully applied. Also, [13] presents an extensive survey of the non-graphical applications where the use of GPU delivers good results.

Recently, the design of GPUs with unified architecture and the development of general-purpose languages which enable the use of the GPU as a generalpurpose coprocessor has renewed and increased the interest in this class of processors. Unfortunately, the rapid evolution of both the hardware and software (programming languages) of GPUs has outdated most of the performance studies available to date.

In this paper, we design and implement a reduced collection of "benchmark" routines, composed of four linear algebra operations (matrix-matrix product, matrix-vector product, saxpy, and scaling of a vector) and an image processing kernel (convolution filter). These routines are employed to evaluate the impact of the improvements introduced in the new generation of GPUs (Nvidia G80), comparing the results with those obtained on a GPU from a previous generation (Nvidia NV44) and current general-purpose processors (AMD Athlon XP 2400+ 
and Intel Core 2 Duo). The ultimate purpose of this evaluation is to characterize the properties that need to be present in an algorithm so that it can be correctly and efficiently adapted into the GPU execution model.

The rest of the paper is organized as follows. Section 2 describes the basic architecture and execution model of both the old and new generations of GPUs, illustrates the main differences between them, and points out the improvements introduced in the latter. Section 3 characterizes the routines in the benchmark collection. Section 4 evaluates the performance of the benchmark routines on the Nvidia NV44, compares the results with those obtained on a CPU, and identifies a set of properties that must be present in an algorithm to deliver high performance on that GPUs. In Section 5 we repeat the study using now the Nvidia G80 as the execution platform. In particular, in that section we evaluate the impact of the improvements introduced in the memory hierarchy, interconnection buses, etc., in the performance of the benchmark routines. Finally, Section 6 summarizes the conclusions that can be extracted from our analysis.

\section{GPU Architecture and Execution Model}

\subsection{GPU graphics pipeline}

The graphics pipeline consists of a set of sequential stages, each one with a specific functionality and operating on an specific type of data. The process transforms original graphical information (vertices) into data suitable for being shown on display (pixels). Figure 1 illustrates the usual stages (or phases) that form the graphics pipeline.

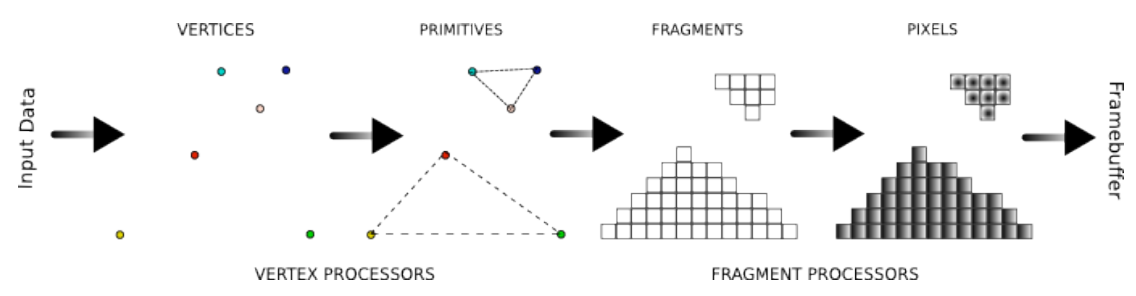

Fig. 1. Graphics pipeline process with its main stages.

Current GPUs implement this pipeline depending on the generation they belong to. Thus, classical GPUs have specific hardware units, known as shaders (or processors), for each one of the stages of the graphics pipeline. On the other hand, GPUs from the latest generation have a unified shader (or unified processor), with the ability to both execute any of the stages of the pipeline and work with any type of graphical data. 


\subsection{Classical architecture}

Until 2006 GPUs were based on a design where each pipeline stage was executed on a specific hardware unit or processor inside the pipeline. Thus, e.g., vertices are processed by vertex processors while pixels (also called fragments) are transformed by fragment processors. In practice, general-purpose algorithms implemented on these classical architectures exploit fragment processors only, due to their larger number and broader functionality. Fragment processors operate in SIMD mode, taking a fragment as input, and processing its attributes; they can also process vectorial data types, working simultaneously on the four components of a fragment (R, G, B, and A). This class of hardware is able to read from random memory locations (commonly known as a gather operation in graphics algorithms), but can only modify one memory position per processed fragment, the one associated with the position of the fragment. This lack of support for scatter is one of the main restrictions of the classical GPU.

In the latter generations of this "classical architecture", programming capabilities were added to vertex and fragment processors. Altogether, the previous characteristics enable the use of fragment processors as a hardware platform to process non-graphical data. Unfortunately, the graphical-oriented design of this class of hardware, its SIMD execution model, and the lack of a sophisticated memory hierarchy are problems for an efficient implementation of generalpurpose applications on the GPU.

In particular, one of the major obstacles when adapting general-purpose algorithms to this class of processors lies in the implementation. Usually, specific languages as Cg or GLSL are used [4,14], together with graphical libraries like OpenGL or DirectX $[16,9]$. However, these programming tools require a deep

knowledge of the target language as well as the underlying architecture in order to obtain correct results and high performance.

\subsection{Unified architecture}

In 2006 a new generation of GPUs was introduced, with a completely different architectural design. These new platforms feature a unified architecture, with one processing unit or unified shader that is able to work with any kind of graphical data, transforming the sequential pipeline in Figure 1 into a cyclic one, in which the behavior of the unified shader varies depending on the stage of the pipeline that it is being executed at each moment.

There are several characteristics in the new generation of GPUs which specifically favor their use as a general-purpose coprocessor: in general, the clock frequency of the unified shader is much higher than that of a fragment processor (even though it is still much lower than the clock frequency of current CPUs); the shader consists of a large collection of computation units (up to 128, depending on the GPU version), called Streaming Processors (SPs), which operate in clusters of 16 processors in SIMD mode on the input data stream; and the architecture includes a sophisticated memory hierarchy, which comprises a L2 cache and small fast memories shared by all the SP in the same cluster. 
These hardware advances are complemented with the CUDA [2] generalpurpose programming library, which eases the programming effort on these platforms. In fact, CUDA has been proposed as a standard (although only compatible with Nvidia hardware) to program the new generation of GPUs, without the requirement of learning more complex graphics-oriented languages.

\section{Benchmark Collection}

In order to identify the algorithmic properties that yield correct and efficient codes for the GPU execution model, we have studied three major computational aspects of algorithms:

Data parallelism. The replication of functional units inside the GPU (fragment processors in the non-unified architectures, SPs in the unified architectures) makes this class of architectures specially appropriate for applications which exhibit a high degree of data parallelism.

Input data reutilization. The simple memory hierarchy in non-unified GPUs makes it difficult to exploit the locality of reference; in these architectures, high memory latency and limited bus bandwidth imply a penalty cost much higher than in a CPU; for this reason, input data reutilization is one of the biggest issues when trying to attain high performance on graphics processors.

Computational intensity per stream element. Due to the previous restriction, to achieve high performance the expensive cost of memory references should be masked with a high number of operations per memory access.

Our benchmark collection is composed of four Basic Linear Algebra Subprograms or BLAS [5]: the matrix-matrix product (SGEMM), the matrix-vector product (SGEMV), the "saxpy" (SAXPY), and the scaling of a vector (SSCAL); and a convolution filter, common in image processing. From the computational viewpoint, the routines in the benchmark present the following properties:

SGEMM features some properties that make it a good candidate to achieve good results when mapped into graphics hardware. It exhibits a regular memory access pattern, a high degree of data parallelism, and a very high computational load. On the other side, it is interesting to study the importance of the high input data reutilization in this type of algorithm.

SGEMV exhibits a smaller input data reutilization than SGEMM. Thus, while each input element for the SGEMM routine is used $O(n)$ times to compute the result, SGEMV only reutilizes $O(n)$ times the data of the input matrix but $O(1)$ times the data of the vector. This behavior makes the matrix-vector product routine a more streaming-oriented code, and so it is theoretically possible to achieve better results on a GPU.

SAXPY and SSCAL are specially interesting for graphics processors, as they do not reutilize input data at all. This type of algorithms are fully streamoriented, and so they are ideal to be executed on a GPU. 
The main difference between these two operations, from the performance viewpoint, is the amount of computational load per stream element. Thus, SAXPY performs twice as many operations as SSCAL per element. This difference offers some information on the importance of the computational load in the performance of the processor.

Convolution. These filters exhibit some properties which favor GPU hardware. First, the high degree of data parallelism will take advantage of fragment processors (or SP) replication of modern GPUs. Second, input data reutilization is very low (proportional to the size of the applied filter, usually small). Third, the computational load per calculated element is high, and based on multiply-and-add (MAD) operations, for which the GPU is specially appropriate.

Table 1 summarizes the computational aspects of the routines in the benchmark.

\begin{tabular}{|c|c|c|}
\hline Routine & BLAS & Properties \\
\hline \multirow{3}{*}{ SGEMM } & \multirow{3}{*}{ BLAS-3 } & High data parallelism \\
\hline & & High computational load per element \\
\hline & & High input data reutilization $O(n)$ \\
\hline \multirow{3}{*}{ SGEMV } & \multirow{3}{*}{ BLAS-2 } & High data parallelism \\
\hline & & High computational load per element \\
\hline & & Low input data reutilization $O(n) / O(1)$ \\
\hline \multirow{3}{*}{ SAXPY } & \multirow{3}{*}{ BLAS-1 } & High data parallelism \\
\hline & & Low computational load per element \\
\hline & & No input data reutilization \\
\hline \multirow{3}{*}{ SSCAL } & \multirow{3}{*}{ BLAS-1 } & High data parallelism \\
\hline & & Very low computational load per element \\
\hline & & No input data reutilization \\
\hline \multirow{3}{*}{ Convolution } & \multirow{3}{*}{-} & High data parallelism \\
\hline & & High computational load per element \\
\hline & & Low input data reutilization \\
\hline
\end{tabular}

Table 1. Summary of the computational aspects of the routines in the benchmark.

All implementations of these routines operate on single-precision floatingpoint data in our experiments. Only square matrices of the same dimension were employed in the evaluation. The performance of the routines was measured in terms of MFLOPs, or millions of floating-point arithmetic operations per second.

\section{Previous Generation GPU-CPU Comparison}

\subsection{Experimental setup}

In this first experiment, we have chosen two experimental platforms of the same generation, an AMD AthlonXP $2400+$ CPU and a Nvidia NV44 GPU processor 
(both from year 2004), so that we can do a fair comparison between generalpurpose and graphics processors. Details on these architectures are given in Table 2. The GNU gcc 4.1.2 compiler is employed in the evaluation.

\begin{tabular}{lll} 
& CPU & GPU \\
\hline Processor & AMD AthlonXP 2400+ & Nvidia GeForce 6200 \\
Codename & Thoroughbred A & NV44A \\
Clock frequency & $2 \mathrm{GHz}$ & $350 \mathrm{MHz}$ \\
Memory speed & $2 \times 133 \mathrm{MHz}$ & $2 \times 250 \mathrm{MHz}$ \\
Peak performance & $8 \mathrm{GFLOPS}$ & $11.2 \mathrm{GFLOPS}$ \\
Bus width & $64 \mathrm{bits}$ & $64 \mathrm{bits}$ \\
Max. bandwidth & $2.1 \mathrm{~GB} / \mathrm{s}$ & $4 \mathrm{~GB} / \mathrm{s}$ \\
Memory & $512 \mathrm{MB}$ DDR & $128 \mathrm{MB}$ DDR \\
Bus Type & \multicolumn{2}{c}{ AGP 8x (2 GB/s transfer rate $)$} \\
Year & $2004 \quad 2004$ \\
\hline
\end{tabular}

Table 2. Description of the hardware used in our first experimental study.

\subsection{Implementation details}

The highly tuned implementation of linear algebra kernels in Goto BLAS 1.15 [7] was used to evaluate the performance of the CPU. No special effort was made to optimize the convolution filter implementation on the CPU other than feeding the appropriate optimization flags to the compiler.

On the other hand, the GPU was programmed using OpenGL and the $\mathrm{Cg}$ language (version 1.5). The routines were adapted to the architecture of the Nvidia NV44 in order to optimize performance, as is briefly described next.

For routine SGEMM, we start from a simple implementation, applying successive refinements in pursue of high performance. First, we adapt the original algorithm using the vectorial capabilities of the fragment processors, as proposed in [3]. This type of optimization usually yields a four-fold increase in performance, and is frequently applied to all types of GPU codes. In addition, we try to exploit the simple cache hierarchy of the Nvidia NV44 by implementing a multipass algorithm, following the ideas in [12]. The goal here is analogous to blocking techniques for CPUs; however, this technique often delivers poorer results on GPUs as the multiple memory writes after each rendering pass penalize the global performance. In general, an SIMD architecture attains higher performance when the instructions are executed only once on the data stream.

We have also implemented simple versions of routines SGEMV, SAXPY, and SSCAL (translated directly from the corresponding CPU algorithms), and optimized ones which exploit the vectorial capabilities of the GPU by applying analogous optimizations to those described above for routine SGEMM.

Convolution filters allow us to introduce simple but powerful optimizations starting from a basic implementation. Our proposal to achieve high performance 
when executing this operation on a GPU is to divide the original $N \times N$ image into four $N / 2 \times N / 2$ quadrants. (For simplicity, we assume here that $N$ is a multiple of 2.) We then map the $(i, j)$ elements of the four quadrants onto the four channels (R, G, B, and A) of an $N / 2 \times N / 2$ data structure. Since a GPU can process four-channel tuples as a scalar element, we can get up to four times higher performance with this type of optimization. Figure 2 illustrates the process. Although this strategy is quite simple, it illustrates the type of optimizations that can be easily applied when implementing general-purpose algorithms on a GPU.

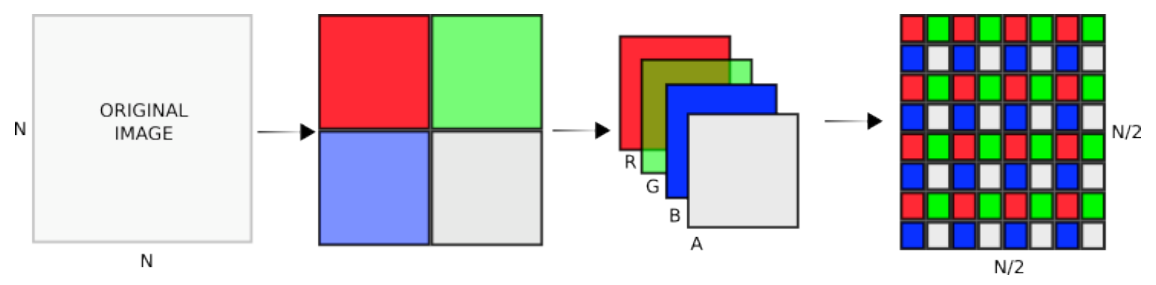

Fig. 2. Optimization applied to the computation of a convolution filters on a GPU with a classical architecture.

\subsection{Experimental results}

We next report the results from our experimental evaluation. By analyzing these, the goal to determine which algorithmic properties (computational aspects in Table 1) favor the execution of an algorithm on a GPU with a classical architecture.

Figure 3 shows the results for routines SGEMM and SGEMV on the CPU and GPU. On the latter architecture, we report two different MFLOPs rates, labeled as "GPU" / "GPU w. TX", obtained respectively by measuring only the execution time on the GPU or timing also the period required to transfer data and results between RAM and video memory. The high input data reutilization of the matrix-matrix product (see left-hand side plot) explains why the routine in Goto BLAS, which exploits the sophisticated cache hierarchy of the AMD CPU, outperforms the GPU implementation by a factor up to 4 . The righthand side plot illustrates how, when the data reutilization is lower as, e.g., in the matrix-vector product, the difference in performance between the CPU and GPU routines decreases, though still favors the CPU (between two and three times higher MFLOPs rate on this architecture).

The figure also reports that the impact of the data transference, however, is less important for routine SGEMM, which carries out a higher computational load per element that is transferred through the bus.

From the previous results, it is possible to conclude that the amount of data reutilization is an important factor in order to achieve high performance on a GPU. Therefore, one could expect that BLAS-1 operations (SAXPY and SSCAL) 

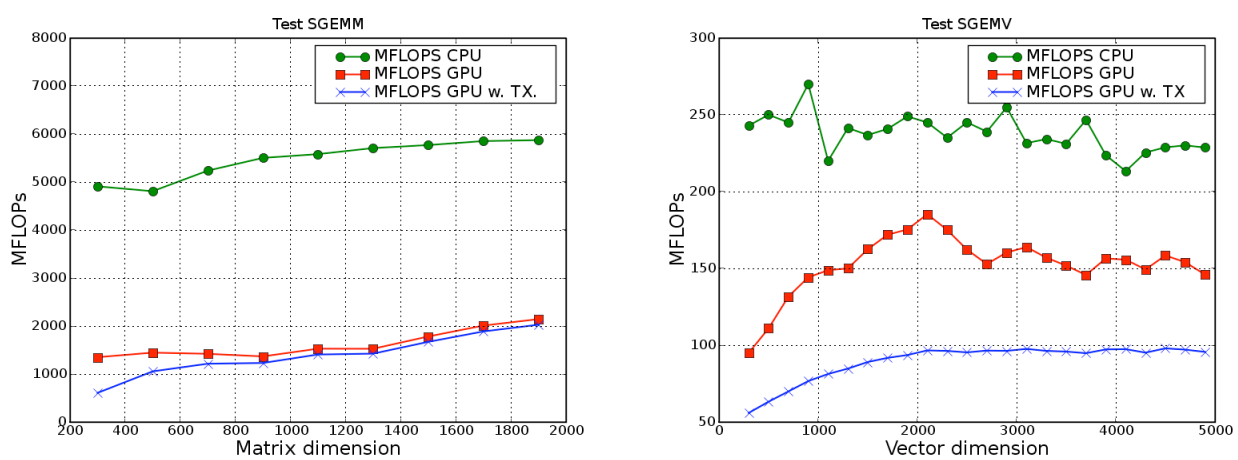

Fig. 3. Performance of routines SGEMM (left-hand side) and SGEMV (right-hand side) on the AMD AthlonXP $2400+$ CPU and the Nvidia NV44 GPU.

will deliver a high MFLOPs rate on this class of hardware. Surprisingly, as shown in Figure 4, we get a poor performance for our implementations of SAXPY and SSCAL, much lower than those of the corresponding CPU implementations.

This behaviour can be explained as follows: the scarce amount of computational load per memory access in BLAS-1 operations limits their performance. This is partially due to the lower efficiency of the memory system of the Nvidia NV44 GPU, with a poor use of cache memories. The elaborated cache memory of the CPU, and its efficient use by the optimized routines in Goto BLAS, are the reasons for such a notable difference in efficiency. Furthermore, results on the GPU are slightly better for SAXPY when compared with the corresponding implementation on CPU than for SSCAL, as the computational load per stream element calculated in the former operation is twice as high as that of SSCAL.

In conclusion, high computational load per stream element is one of the basic conditions for an algorithm to deliver high performance when executed on GPU. Once more, the data transfer stage is very relevant for this type of algorithm, as shown in Figure 4.

Convolution filters combine in the same operation a set of very favorable properties for GPUs: high data parallelism, low input data reutilization, and high computational load per stream element. Figure 5 shows the results of the implementations of the convolution filer on the CPU and GPU. For the latter, we include a simple implementation and a tuned one, with the optimization described at the end of Section 4.

The basic implementation (labeled as GPU) can already improve the performance of the same implementations on the CPU. The optimized implementation (labeled as GPU4) employs the four channels of each element of the input stream in order to store data, attaining a speed-up factor close to $4 \mathrm{x}$ with respect to the basic implementation.

Convolution filters are the type of algorithms that better fit into the execution model of GPUs with classical architecture. These operations exhibit all 

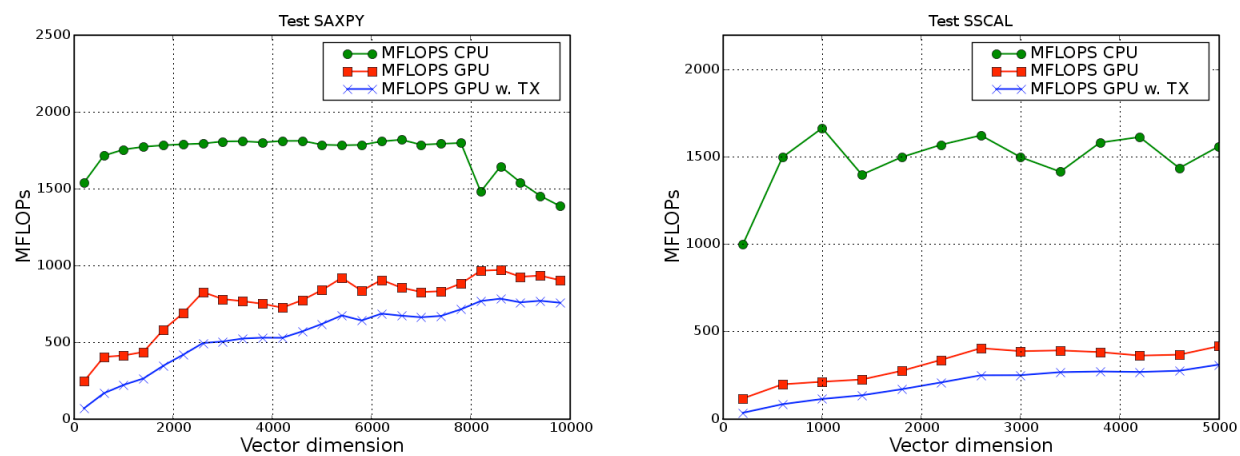

Fig. 4. Performance of routines SAXPY (left-hand side) and SSCAL (right-hand side) on the AMD AthlonXP $2400+$ CPU and the Nvidia NV44 GPU.

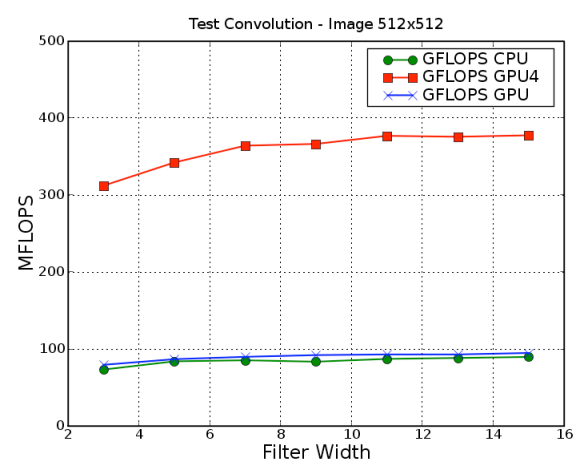

Fig. 5. Performance of the implementations of the convolution filter on the $A M D$ AthlonXP 2400+ CPU and the Nvidia NV44 GPU. 
the properties that make good use of GPUs computational power and, at the same time, hide those aspects in which CPUs are better than graphics processors (basically at memory access).

\section{New Generation GPU-CPU Comparison}

\subsection{Comparison goals}

Although the study of the non-unified generation of GPUs has identified some of the characteristics desirable in algorithms that target GPUs with classical architecture, it is also interesting to carry over this study to new generation GPUs with unified architecture. The goal of this study is to verify if our previous insights also hold for these new architectures, and to evaluate how the hardware and software improvements (at computational power, memory hierarchies and interconnection buses level) affect the performance of the implemented routines.

\section{$5.2 \quad$ Experimental setup}

In this second experiment, we again chose two experimental platforms from the same generation, an Intel Core 2 Duo CPU and a Nvidia GeForce 8800 Ultra (with a Nvidia G80 processor) GPU (year 2007); see Table 3 for details. The GNU gcc 4.1.2 compiler is employed in the evaluation. The multithreading capabilities of Goto BLAS were enabled so that the two cores in the Intel CPU cooperate in solving the linear algebra operations.

The implementations of the linear algebra routines in the the CUBLAS library ([1]) were used in the evaluation. This is a library developed by Nvidia, implemented on top of CUDA, and optimized for unified graphics architectures as the Nvidia G80. The experimental evaluation showed that the implementations in CUBLAS outperformed our implementations using Cg.

\begin{tabular}{lll} 
& CPU & GPU \\
\hline Processor & Intel Core 2 Duo & Nvidia GeForce 8800 Ultra \\
Codename & Crusoe E6320 & G80 \\
Clock frequency & $1.86 \mathrm{GHz}$ & $575 \mathrm{MHz}$ \\
Peak performance & $14.9 \mathrm{GFLOPS}$ & $520 \mathrm{GFLOPS}$ \\
Memory speed & $2 \times 333 \mathrm{MHz}$ & $2 \times 900 \mathrm{MHz}$ \\
Bus width & $64 \mathrm{bits}$ & $384 \mathrm{bits}$ \\
Max. bandwidth & $5.3 \mathrm{~GB} / \mathrm{s}$ & $86.4 \mathrm{~GB} / \mathrm{s}$ \\
Memory & $1024 \mathrm{MB}$ DDR & $768 \mathrm{MB}$ DDR \\
Bus & \multicolumn{2}{c}{ PCI Express x16 $(4 \mathrm{~GB} / \mathrm{s}$ transfer rate $)$} \\
Year & 2007 & 2007 \\
\hline
\end{tabular}

Table 3. Description of the hardware used in our second experimental study. 
For the convolution filter, we implemented a tuned version using CUDA, with intensive use of the fast shared memory per group of SP in order to optimize performance. We also applied other optimizations proposed in [2].

\subsection{Experimental results}

The goal of the following experiments is to determine the algorithmic properties which favor the execution of an algorithm on a GPU with a unified architecture.

Figure 6 shows the performance of routine SGEMM on both platforms. Although this is not the most appropriate algorithm for the GPU (indeed, it only delivers about $20 \%$ of the peak power of the GPU), the performance on that platform is roughly 10 times higher than that obtained on the CPU.

The impact of the transfer time is larger for the unified architecture compared with non-unified architecture, with less powerful interconnection buses. In fact, the peak performance of the Nvidia G80 is about 20 times higher than that of the Nvidia NV44, but the speed of the interconnection bus in the unified platform is only twice as fast as the one in the non-unified platform. This is a major bottleneck in current graphics platforms, and determines that GPU algorithms must be redesigned to reduce the communications so that data in video mamory is reused as much as possible before sending them back to RAM.
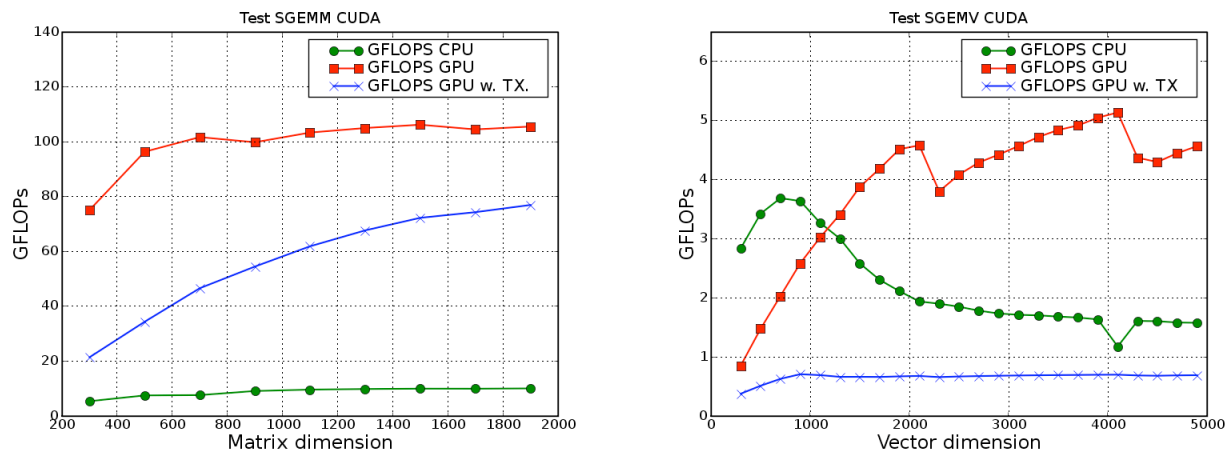

Fig. 6. Performance of routines SGEMM (left-hand side) and SGEMV (right-hand side) on the Intel Core 2 Duo CPU and the Nvidia G80 GPU.

The impact of this bottleneck in the performance of a routine is higher when its computational load decreases. For example, Figure 6 illustrates the performance of routine SGEMV. The decrease in the GFLOPS rate is higher in this case when the transmission time is included. This difference is so important for this routine that, in case the transfer time is considered in the evaluation, the performance is lower on the GPU than on the CPU. When transfer times are not considered, the implementation on the CPU outperforms the CUBLAS im- 
plementation for large stream dimensions. For small streams, the cache memory plays a very important role, , and results are better on the CPU.

Comparing routines SGEMM and SGEMV, the introduction of a sophisticated memory hierarchy in the Nvidia G80 diminishes the impact of the data reutilization. The results for routine SGEMM are better when we compare them with CPU implementation than the results we obtain for routine SGEMV. The introduction of cache memories is one of the main differences between both generations of GPU and, from the previous results, we can conclude it has an important influence in the performance of general-purpose algorithms on GPUs with unified architectures.

The amount of computational load per stream element is also critical in this class of architectures. Figure 7 reports the results for routines SAXPY and SSCAL. The behaviors are similar to those obtained for a GPU with a classical pipeline. Despite being stream-oriented algorithms, without any type of input data reutilization, the results are not comparable with those obtained by the tuned implementations in CUBLAS. The transfer time is more relevant in this case, as the computational load of the algorithms is quite low.
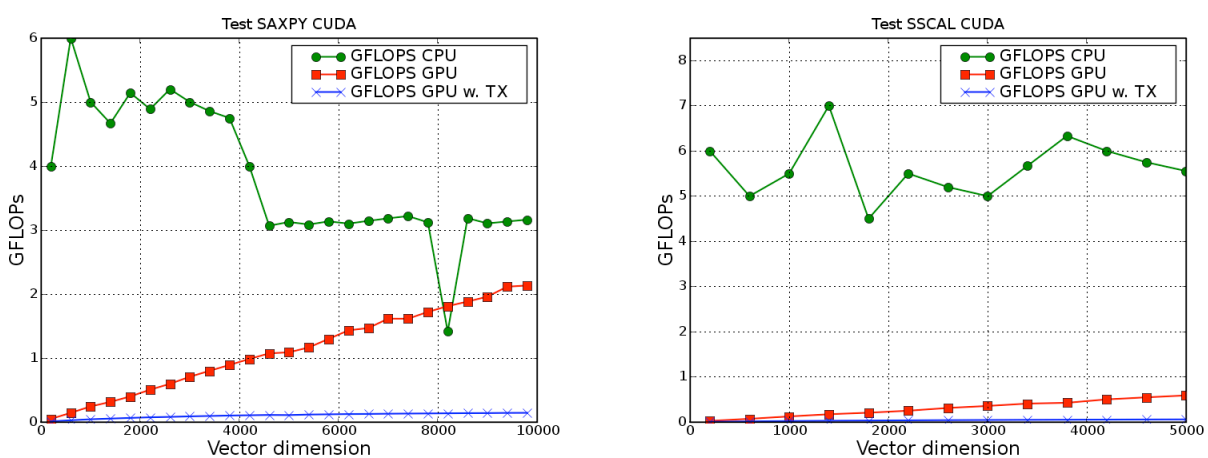

Fig. 7. Performance of routines SAXPY (left-hand side) and SSCAL (right-hand side) on the Intel Core 2 Duo CPU and the Nvidia G80 GPU.

Figure 8 shows the results obtained for the application of a convolution filter on a $512 \times 512$ image and variable filter size. This application again presents the most favorable properties for its execution on current GPU architectures, attaining results up to 20 times better than those achieved for the same routines on a CPU.

\section{Conclusions}

We have presented an study of the properties which favor efficient execution of general-purpose algorithms on a graphics processor, considering both classical and unified architectures. 


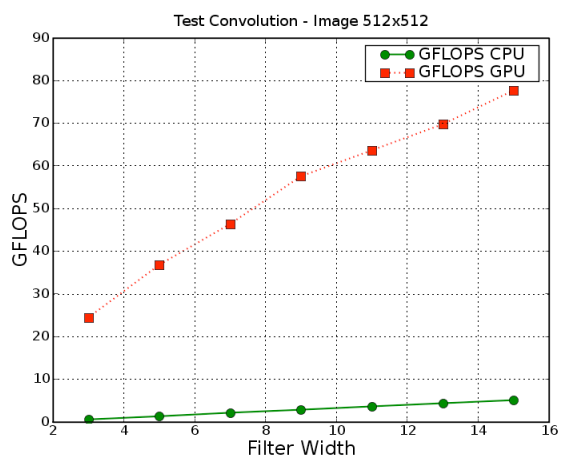

Fig. 8. Performance of the implementations of the convolution filter on the Intel Core 2 Duo CPU and the Nvidia G80 GPU.

GPUs from previous generations, with classical architecture, are suitable for certain types of general-purpose algorithms with three basic characteristics: low input data reutilization, high data level parallelism, and high computational load per stream element. Despite their high computational power, the graphicsoriented nature of this class of hardware carries a set of limitations at the architecture level which ultimately limit the performance of certain types of algorithms like, e.g., routines from BLAS. On the other hand, GPUs of this nature obtain remarkable results for general-purpose algorithms which exhibit the three properties specified above, outperforming in this case the CPU.

The improvements introduced in the new generation of GPU (unified architecture, higher processing units replication, more sophisticated memory hierarchies, etc.) have increased the efficiency of this hardware to execute also for general-purpose algorithms. In fact, current GPUs deliver higher performance than that of timely CPUs in many applications.

Therefore, the last generation of GPUs appears as a high performance and low cost co-processing platform for a larger variety of applications. The emergence of general-purpose languages that facilitate their programming makes them even more interesting hardware from general-purpose computations. Nevertheless, GPUs still present some limitations in general-purpose computing such as numerical precision, data transfer stages, memory hierarchies not as sophisticated as CPU ones, etc. All this makes necessary to evaluate carefully the suitability of GPU as an accelerator for calculations.

\section{Acknowledgments}

This work has been supported by the CICYT project TIN2005-09037-C02-02 and FEDER. Francisco Igual-Peña is supported as well by a research fellowship from the Universidad Jaume I of Castellón (PREDOC/2006/02). 


\section{References}

1. NVIDIA Corp. NVIDIA CUBLAS Library. 2007.

2. NVIDIA Corp. NVIDIA CUDA Compute Unified Device Architecture. Programming Guide. 2007.

3. K. Fatahalian, J. Sugerman, and P. Hanrahan. Understanding the efficiency of GPU algorithms for matrix-matrix multiplication. Graphics Hardware, 2004.

4. R. Fernando. The Cg Tutorial: The Definitive Guide to Programmable Real-Time Graphics. 2004.

5. Basic Linear Algebra Subprograms Technical (BLAST) Forum. Basic Linear Algebra Subprograms Technical (BLAST) Forum Standard. 2001.

6. N. Galoppo, N. Govindaraju, M. Henson, and D. Monocha. LU-GPU: Efficient algorithms for solving dense linear systems on graphics hardware. In $A C M / I E E E$ SC-05 Conference, 2005.

7. K. Goto and R. Van de Geijn. High-performance implementation of the level-3 BLAS. ACM Transactions on Mathematical Software.

8. N. Govindaraju, B. Lloyd, W. Wang, M. Lin, and D. Manocha. Fast computation of database operations using graphics processors. Proceedings of the 2004 ACM SIGMOD International Conference on Management of Data, pages 215-226, June 2004.

9. K. Gray. Microsoft DirectX 9 Programmable Graphics Pipeline. 2005.

10. J.Y. Hong and M.D. Wang. High speed processing of biomedical images using programmable GPU. In Image Processing, 2004. ICIP '04. 2004 International Conference on, volume 4, pages 2455 - 2458 Vol. 4, 24-27 Oct. 2004.

11. E.S. Larsen and D. McAllister. Fast matrix multiplies using graphics hardware. In Supercomputing, ACM/IEEE 2001 Conference, pages 43 - 43, Nov. 2001.

12. A. Moravánszky. Dense matrix algebra on the GPU. 2003.

13. J.D. Owens, D. Luebke, N. Govindaraju, M. Harris, J. Krüger, A.E. Lefohn, and T.J. Purcell. A survey of general-purpose computation on graphics hardware. Computer Graphics Forum, 2007.

14. R.J. Rost, J. Kessenich, and B. Lichtenbelt. OpenGL Shading Language. 2004.

15. A. Ruiz, O. Sertel, M. Ujaldon, U. Catalyurek, J. Saltz, and M. Gurcan. Pathological image analysis using the GPU: Stroma classification for neuroblastoma. Proceedings IEEE Intl. Conference on BioInformation and BioMedicine, 2007.

16. M. Segal and K. Akeley. The OpenGL Graphics System: A Specification. 2006. 